\title{
Heterogeneous Catalysis: Enabling a Sustainable Future
}

\author{
Xijun $\mathrm{Hu}^{1 *}$ and Alex C. K. Yip ${ }^{2}$ \\ ${ }^{1}$ Department of Chemical and Biological Engineering, The Hong Kong University of Science and Technology, Hong Kong, \\ China, ${ }^{2}$ Laboratory for Energy and Environmental Catalysis, Department of Chemical and Process Engineering, The \\ University of Canterbury, Christchurch, New Zealand
}

Keywords: heterogeneous catalysis, grand challenges, sustainability, hydrogen energy, greenhouse gas emission

\section{GRAND CHALLENGES FOR HETEROGENEOUS CATALYSIS}

Over the past decade, catalysis scientists have been at the forefront in addressing various challenges in climate change, pollution, and sustainable energy. The ever-changing landscape in addressing these complex and intricately-linked challenges saw many innovative strategies and approaches being developed by the catalysis community.

At the heart of catalysis science, heterogeneous catalysis drives many critical industrial processes because of the excellent capabilities of accelerating the rate of reactions with low cost, high conversion, and product selectivity. The recyclability nature of heterogeneous catalysts enables green and sustainable manufacturing and avoids the secondary pollution problem. To date, heterogeneous catalysts play an important part in producing more than $80 \%$ of all chemical products in the world. In 2019, the global catalyst market size was valued at USD 33.9 billion and was expected to grow at a compound annual growth rate of 4.4\% from 2020 to 2027.

OPEN ACCESS

Edited by:

Frank Hollmann,

Delft University of

Technology, Netherlands

Reviewed by:

Felix Studt,

Karlsruhe Institute of Technology

(KIT), Germany

*Correspondence:

Xijun $\mathrm{Hu}$

kexhu@ust.hk

Specialty section:

This article was submitted to Heterogeneous Catalysis,

a section of the journal

Frontiers in Catalysis

Received: 14 February 2021

Accepted: 25 February 2021

Published: 18 March 2021

Citation:

Hu X and Yip ACK (2021) Heterogeneous Catalysis: Enabling a Sustainable Future.

Front. Catal. 1:667675.

doi: 10.3389/fctls.2021.667675
Rising demand for catalysts is evidenced by pillared industries, including energy, environmental protection, fine chemical synthesis, and bulk chemical production, etc. It is an undeniable fact that every breakthrough made in the field of heterogeneous catalysis led to economic success for the major world economies and improved people's everyday lives.

The heterogeneous catalysis community is instrumental for grand global challenges in energy and the environment at a high level. The year 2020 with COVID-19 marked a new normal to our world. We saw fewer cars on the roads, canceled flights, restaurants' shutdown, unprecedented restrictions on everyday activities, etc. Despite putting our economy to a halt, we used less carbon-based fuel and potentially released fewer pollutants to the environment. But what does this mean to the post-COVID-19 world?

The overall greenhouse gas (GHG) emission in 2020 was $\sim 4 \%$ less than the previous year. While this interim result sounds positive environmentally, the $4 \%$ reduction in GHG emission will only contribute to about two-thirds of what is required to meet the annual " $1.5^{\circ} \mathrm{C}$ target" (that is, to keep the global temperature rise below $1.5^{\circ} \mathrm{C}$ by the end of this century). The implication is that minimizing human activities, such as restricted air travels, mass gathering, and use of petrolpowered vehicles, even to a level as strict as what we are going through in the COVID pandemic, is not sufficient to solve the climate change problem. This event confirms that a more pragmatic approach is needed.

For the years to come, the holy grail of the environment is still developing sustainable energy that replaces fossil fuels on a significant scale. The challenges include generating renewable fuel, securing solar power and wind energy, production of sustainable electricity, and safe and efficient long-term energy storage (Gong and Luque, 2014). The cost of harvesting sustainable energy was traditionally high compared to fossil fuels. Driven by the state-of-the-art engineering (e.g., wind turbines with much higher capacity factors than a decade ago) and policies such as tax credits, we saw the generation cost of renewables dropped in the past 5 years. In many places, renewables' cost 
is comparable or even below that of conventional energy sources such as oil, natural gas, and coal. Hydroelectric power is now the cheapest source of renewable energy. It is averaged at US\$ 0.05 per kilowatt-hour $(\mathrm{kWh})$ and the cost of offshore wind was close to US $\$ 0.13$ per kWh as of May 2019. Before COVID-19, these renewable options started to become economically viable compared to the cost of fossil fuels, typically ranging from US $\$ 0.05$ to over US $\$ 0.15$ per $\mathrm{kWh}$. The abovementioned positive incentives imply that our research in complementary areas to renewables, such as catalytic biomass conversion, hydrogen generation/storage, and $\mathrm{CO}_{2}$ capture/utilization, will remain critical for the world in the future.

Hydrogen is regarded as clean energy and chemical feedstock, which can be used for fuel cells, transport fuel, oil refining, and urea production. However, over $95 \%$ of the hydrogen currently produced is derived from fossil fuels, primarily through steam methane reforming. Hydrogen can be produced from renewable electricity through catalytic water electrolysis or water-gas-shift reaction, but commercial production has challenges in scaleup with high demand for electricity (Dawood et al., 2020). $\mathrm{CO}_{2}$ sequestration for mitigation of GHG emission has been studied for many years, and, again, increased costs and increased risk for $\mathrm{CO}_{2}$ leakage hinder large-scale implementation. $\mathrm{CO}_{2}$ sequestration is also a waste of potentially valuable chemical feedstock $\left(\mathrm{CO}_{2}\right)$. We foresee the catalysis community will have a strong focus on developing new catalytic processes and new catalysts to produce bio-hydrogen from renewable energy sources and capture $\mathrm{CO}_{2}$ effectively.

Scientifically, a successful heterogeneous catalytic reaction is governed by three fundamental elementary steps, namely adsorption, surface reactions, and desorption, associated with a solid surface. As such, the advancement of heterogeneous catalysis is inseparable from the knowledge of surface and interface science, entailing solutions to grand challenges of three main areas: catalyst design and synthesis, in situ characterization, and understanding catalysis complexity.

Catalyst design and synthesis is the art of choosing the right active component to produce the desired chemistry in catalysis. Precise control of catalytic components on catalyst support requires sophisticated synthesis techniques and science to scale up and satisfy practical manufacturing. A classic example would be the synthesis of higher alcohol from syngas, which requires two distinct metal sites in a close molecular distance on a surface to enable a synergistic catalytic effect (Khan et al., 2020). Integration of multiple catalysts in a single environment also plays a vital part in process intensification.

On the other hand, purpose-built microporous materials, such as zeolites, metal-organic frameworks (MOFs), or covalent organic frameworks (COFs), are important to industrial applications. Taking zeolites as an example, while their catalytic properties mainly rely on the high surface area and cationexchange capacity, zeolite catalysts usually require multiple functionalities, such as pore size/shape, tunable acidity, hydrophobicity/hydrophilicity, hydrothermal stability, and specifically, good resistance to coke deactivation. Hierarchical zeolites and their designs provide a solution to satisfy such requirements. New synthesis strategies of hierarchical zeolite, which are sustainable, environmentally friendly and costeffective, are essential areas of study in modern catalysis (Jia et al., 2018).

In the past, catalyst characterization was conducted "off-line" from the reaction environment, preventing physical insights from studying catalytic systems under practical conditions. We saw in the last decade a rapid development of in situ/operando characterization tools, which helped researchers to identify the catalyst descriptors and structure-reactivity relations (Choi et al., 2020). As the in situ/operando characterization tools become more sophisticated and functional under harsh conditions, e.g., high temperature and high pressure, we shall see a quantum leap in our ability to unlock many new catalysts and catalytic routes.

Having a fundamental understanding of how surfaces catalyze a chemical reaction is an essential component of heterogeneous catalysis. We can establish predictive modeling strategies for realistic catalysis systems by close-coupling of theory and experiments. Computational methods, such as molecular dynamics simulation, density functional theory (DFT) calculations, will continue to become the fundamental basis for understanding catalysis and surface chemical reactivity. One of the biggest challenges is still lacking a transient view of heterogeneous catalysis that explains out-of-equilibrium processes. The development of computational chemistry methods will produce a toolbox to unravel catalysis complexity and reaction mechanisms under different process scenarios (Gaggioli et al., 2019). Research in this area will shape innovation in catalysis and lead to more sustainable chemical processes.

The international catalysis community needs to share cuttingedge research works with each other proactively in a transparent manner. By making our knowledge more accessible to the world, we will be able to push forward the development of the enabling tools and the engineering foundations needed to address the formidable challenges that we face as we strive to improve everyone's quality of life.

The Heterogeneous Catalysis specialty section within Frontiers in Catalysis aims to capture the recent impactful research works in the field, through which to provide inspiration and stimulation to the community.

\section{AUTHOR CONTRIBUTIONS}

$\mathrm{XH}$ and $\mathrm{AY}$ prepared the draft of the manuscript, revised the manuscript, and read and approved the final manuscript. All authors contributed to the article and approved the submitted version. 


\section{REFERENCES}

Choi, J. J., Kim, T. S., Kim, D., Lee, S. W., and Park, J. Y. (2020). Operando surface characterization on catalytic and energy materials from single crystals to nanoparticles. ACS Nano 14, 16392-16413. doi: 10.1021/acsnano.0c07549

Dawood, F., Anda, M., and Shafiullah, G. M. (2020). Hydrogen production for energy: an overview. Int. J. Hydrogen Energy 45, 3847-3869. doi: 10.1016/j.ijhydene.2019.12.059

Gaggioli, C. A., Stoneburner, S. J., Cramer, C. J., and Gagliardi, L. (2019). Beyond density functional theory: the multiconfigurational approach to model heterogeneous catalysis. ACS Catal. 9, 8481-8502. doi: 10.1021 /acscatal.9b01775

Gong, J., and Luque, R. (2014). Catalysis for production of renewable energy. Chem. Soc. Rev. 43, 7466-7468. doi: 10.1039/C4CS90084G

Jia, X., Khan, W. U., Wu, Z., Choi, J., and Yip, A. C. K. (2018). Modern synthesis strategies for hierarchical zeolites: bottom-up versus top-down strategies. Adv. Powder Technol. 30, 467-484. doi: 10.1016/j.apt.2018. 12.014

Khan, W. U., Baharudin, L., Choi, J., and Yip, A. C. K. (2020). Recent progress in CO hydrogenation over bimetallic catalysts for higher alcohol synthesis. ChemCatChem 13, 111-120. doi: 10.1002/cctc.202001436

Conflict of Interest: The authors declare that the research was conducted in the absence of any commercial or financial relationships that could be construed as a potential conflict of interest.

Copyright (c) $2021 \mathrm{Hu}$ and Yip. This is an open-access article distributed under the terms of the Creative Commons Attribution License (CC BY). The use, distribution or reproduction in other forums is permitted, provided the original author $(s)$ and the copyright owner(s) are credited and that the original publication in this journal is cited, in accordance with accepted academic practice. No use, distribution or reproduction is permitted which does not comply with these terms. 